TRANSACTIONS OF THE

AMERICAN MATHEMATICAL SOCIETY

Volume 351, Number 12, Pages 4753-4767

S 0002-9947(99)02556-8

Article electronically published on August 25, 1999

\title{
CAPACITY CONVERGENCE RESULTS AND APPLICATIONS TO A BERSTEIN-MARKOV INEQUALITY
}

\author{
T. BLOOM AND N. LEVENBERG
}

\begin{abstract}
Given a sequence $\left\{E_{j}\right\}$ of Borel subsets of a given non-pluripolar Borel set $E$ in the unit ball $B$ in $\mathbf{C}^{N}$ with $E \subset \subset B$, we show that the relative capacities $C\left(E_{j}\right)$ converge to $C(E)$ if and only if the relative (global) extremal functions $u_{E_{j}}^{*}\left(V_{E_{j}}^{*}\right)$ converge pointwise to $u_{E}^{*}\left(V_{E}^{*}\right)$. This is used to prove a sufficient mass-density condition on a finite positive Borel measure with compact support $K$ in $\mathbf{C}^{N}$ guaranteeing that the pair $(K, \mu)$ satisfy a Bernstein-Markov inequality. This implies that the $L^{2}$-orthonormal polynomials associated to $\mu$ may be used to recover the global extremal function $V_{K}^{*}$.
\end{abstract}

\section{InTRODUCTION}

Let $E$ be a bounded Borel set with closure $\bar{E}$ contained in a bounded, hyperconvex domain $\Omega$ in $\mathbf{C}^{N}$. This means that there exists a continuous, negative plurisubharmonic (psh) exhaustion function for $\Omega$. We set

$$
U_{E, \Omega}(z):=\sup \{u(z): u \text { psh in } \Omega, u \leq 0, u \leq-1 \text { on } E\}
$$

and call $U_{E, \Omega}^{*}(z):=\lim \sup _{\zeta \rightarrow z} U_{E, \Omega}(\zeta)$ the relative extremal function of $E$ (relative to $\Omega$ ). For most of this paper, we take $\Omega=B$, the unit ball in $\mathbf{C}^{N}$; in this case we use the notation $U_{E}:=U_{E, B}$. Also, letting

$$
V_{E}(z):=\sup \{u(z): u \in L, u \leq 0 \text { on } E\}
$$

where

$$
L:=\left\{u \operatorname{psh} \text { in } \mathbf{C}^{N}: u(z) \leq \log ^{+}|z|+C\right\}
$$

is the class of psh functions of logarithmic growth (here $|z|=\left(\sum_{i=1}^{n}\left|z_{i}\right|^{2}\right)^{1 / 2}$ and $\left.\log ^{+}|z|=\max (0, \log |z|)\right)$, we call $V_{E}^{*}(z):=\lim \sup _{\zeta \rightarrow z} V_{E}(\zeta)$ the global extremal function of $E$. For future use, we also define

$$
L_{+}:=\left\{u: u \text { is psh on } \mathbf{C}^{N} \text { and } \log ^{+}|z|-C \leq u \leq \log ^{+}|z|+C\right\} .
$$

The constants $C$ in the definitions of $L$ and $L_{+}$may depend on $u$.

It is well-known that $U_{E, \Omega}^{*} \equiv 0 \Longleftrightarrow V_{E}^{*} \equiv+\infty \Longleftrightarrow E$ is pluripolar; i.e., there exists $u$ psh in $\mathbf{C}^{N}$ with $E \subset\left\{z \in \mathbf{C}^{N}: u(z)=-\infty\right\}$. If $E$ is not pluripolar, then, using the complex Monge-Ampere operator $\left(d d^{c}(\cdot)\right)^{N}$ for locally bounded psh functions, we can define the relative and global equilibrium measures $\left(d d^{c} U_{E, \Omega}^{*}\right)^{N}$

Received by the editors February 11, 1998 and, in revised form, March 5, 1999.

1991 Mathematics Subject Classification. Primary 31C15, 32F05, 41A17.

(C)1999 American Mathematical Society 
and $\left(d d^{c} V_{E}^{*}\right)^{N}$ for $E$; these measures are supported on $\bar{E}$. We will call a Borel set $E \subset \Omega$ regular if $U_{E, \Omega}^{*}=-1$ on $E$ (equivalently, $V_{E}^{*}=0$ on $E$ ). For $E \subset \Omega$ compact, $E$ is regular if $U_{E, \Omega}$ is continuous. Finally, we say that a property holds quasi-everywhere (q.e.) on a set $S$ if the property holds on all of $S$ except perhaps for a pluripolar set. For example, if $E$ is not pluripolar, then $U_{E, \Omega}^{*}=-1$ q.e. on $E$ (cf., [BT1]).

One can also define a nonnegative function $C(E, \Omega)$ on the Borel subsets $E$ of $\Omega$ via

$$
C(E, \Omega):=\sup \left\{\int_{E}\left(d d^{c} u\right)^{N}: u \text { psh on } \Omega, 0 \leq u \leq 1\right\} .
$$

For Borel sets we have (Proposition 4.7.2[K])

$$
C(E, \Omega)=\int_{\Omega}\left(d d^{c} U_{E, \Omega}^{*}\right)^{N}
$$

moreover, from Proposition 10.1 [BT1] it follows that

$$
C(E, \Omega)=\int_{\Omega}-U_{E, \Omega}^{*}\left(d d^{c} U_{E, \Omega}^{*}\right)^{N}
$$

This set function, which we denote by $C(E)$ if $\Omega=B$, enjoys the properties that

1. if $E_{1} \subset E_{2}$, then $C\left(E_{1}\right) \leq C\left(E_{2}\right)$;

2. if $\left\{K_{j}\right\}$ are compact subsets of $B$ with $K_{j+1} \subset K_{j}, j=1,2, \ldots$, then $C\left(\cap K_{j}\right)$ $=\lim _{j \rightarrow \infty} C\left(K_{j}\right)$;

3. if $\left\{E_{j}\right\}$ are Borel subsets of $B$ with $E_{j+1} \supset E_{j}, j=1,2, \ldots$, then $C\left(\bigcup E_{j}\right)=$ $\lim _{j \rightarrow \infty} C\left(E_{j}\right)$.

This last property is easily seen in the case where $\bigcup E_{j} \subset \subset B$ using the fact that the complex Monge-Ampere operator $\left(d d^{c}(\cdot)\right)^{N}$ is continuous under monotone limits; i.e., if $\left\{u_{j}\right\}$ is a family of locally bounded psh functions on $B$ which either increase or decrease a.e. to a locally bounded psh function $u$, then the Monge-Ampere measures $\left(d d^{c} u_{j}\right)^{N}$ converge weak-* to $\left(d d^{c} u\right)^{N}$ (cf., [BT1]). If $E:=\bigcup E_{j} \subset \subset B$ where $E_{j+1} \supset E_{j}, j=1,2, \ldots$, then the relative extremal functions $u_{j}:=U_{E_{j}}^{*}$ decrease to $u:=U_{E}^{*}$; thus, if $\phi \in C_{0}^{\infty}(B)$ is nonnegative and equal to one on $\bar{E}$,

$$
\lim _{j \rightarrow \infty} C\left(E_{j}\right)=\lim _{j \rightarrow \infty} \int_{B} \phi\left(d d^{c} u_{j}\right)^{N}=\int_{B} \phi\left(d d^{c} u\right)^{N}=C(E) .
$$

We will consider a sequence $\left\{E_{j}\right\}$ of Borel subsets of a given non-pluripolar Borel set $E$ in the unit ball $B$ in $\mathbf{C}^{N}$ but we do not assume the sequence is monotone. One of our results (Theorem 1.1) is that $u_{j} \rightarrow u$ pointwise on $B$ if and only if $\lim _{j \rightarrow \infty} C\left(E_{j}\right)=C(E)$. We also give a related condition on the global extremal functions $V_{E_{j}}^{*}$ and their corresponding Robin functions (the definition will be given in the next section).

Our motivation for this research comes from a study of asymptotic behavior of orthonormal polynomials. Precisely, let $\mu$ be a finite positive Borel measure with compact support $S(\mu):=K$ in $\mathbf{C}^{N}$. We say that the pair $(K, \mu)$ satisfies a Bernstein-Markov inequality if for each $\epsilon>0$ there exists a positive constant $M=M(\epsilon)$ such that

$$
\|p\|_{K}:=\sup _{z \in K}|p(z)| \leq M(1+\epsilon)^{\operatorname{deg} p}\|p\|_{L^{2}(\mu)}
$$


for all holomorphic polynomials $p=p(z)$. From the point of view of pluripotential theory, such an inequality is important because one can recover the global extremal function $V_{K}^{*}$ - under certain additional assumptions on $K$-from the orthogonal polynomials associated to $\mu$. We make this precise in (0.2) below.

For an arbitrary compact set $K$ in $\mathbf{C}^{N}$ we have

$$
V_{K}(z):=\max \left[0, \sup \left\{\frac{1}{\operatorname{deg} p} \log |p(z)|: p \text { holomorphic polynomial, }\|p\|_{K} \leq 1\right\}\right]
$$

(Theorem 5.1.7 [K]). We will use $K, K_{j}$ to denote compact sets while $E, E_{j}$ will be used for Borel sets (compact or not). Suppose now $K$ is a non-pluripolar compact set. Then $\mu_{K}:=\left(d d^{c} V_{K}^{*}\right)^{N}$ is supported in $K$ (cf., [BT1] or [K]); furthermore, if $K$ is regular, then $\left(K, \mu_{K}\right)$ satisfy (0.1) [TZ]. On the other hand, it is known that for any regular compact set $K$ in $\mathbf{C}^{N}$, there exists a discrete measure $\mu$ with support equal to $K$ such that $(K, \mu)$ satisfy $(0.1)$. This phenomenon was observed by Ullman on the interval $[0,1] \subset \mathbf{C}$ in [U]; see [ST] for $N=1$ and [Bl1] for arbitrary $N$.

Given a unisolvent compact set $K$ - this means that if a polynomial $p$ vanishes on $K$ then $p \equiv 0$ - the standard monomials $\left\{z^{\alpha}:=z_{1}^{\alpha_{1}} \cdots z_{N}^{\alpha_{N}}\right\}$ are linearly independent in $L^{2}(\mu)$ (cf., [Bl1], Proposition 3.5 or [L], Proposition 3.1); thus, via the Gram-Schmidt procedure, we obtain orthonormal polynomials $\left\{p_{\alpha}\right\}$. Here we are using the standard multi-index notation $\alpha:=\left(\alpha_{1}, \ldots, \alpha_{N}\right)$ and $|\alpha|:=\alpha_{1}+\ldots+\alpha_{N}$. It follows from [Z] (see also [Bl1]) that if $K$ is regular and $(K, \mu)$ satisfy $(0.1)$, then

$$
\limsup _{|\alpha| \rightarrow+\infty} \frac{1}{|\alpha|} \log \left|p_{\alpha}(z)\right| \leq V_{K}(z)
$$

for all $z \in \mathbf{C}^{N}$ with equality for $z \in \mathbf{C}^{N} \backslash \hat{K}$, where

$$
\hat{K}:=\left\{z \in \mathbf{C}^{N}:|p(z)| \leq\|p\|_{K} \text { for all polynomials } p\right\}
$$

denotes the polynomial hull of $K$.

From the existence of a discrete measure $\mu$ with $K:=S(\mu)$ regular such that $(K, \mu)$ satisfies $(0.1)$, we see that it is not necessary for the carriers of $\mu$ to be "thick" in order for (0.1) to hold (a carrier of $\mu$ is a Borel subset of $S(\mu)$ of full measure); indeed, in [ST] $(N=1)$ and [B11] it is shown that a type of "massdensity" condition on $\mu$ suffices to ensure (0.1). We recall the result in [Bl1] in Theorem 2.1 below. One goal in this note is to discuss, in section 2, more general mass-density conditions on measures guaranteeing that (0.1) holds (Theorem 2.2); here we utilize the capacity convergence results of section 1 . We end the paper in section 3 with some interesting open questions in these topics.

The second author would like to thank E. Bedford, U. Cegrell, S. Kolodziej, E. Poletsky and Y. Xing for helpful discussions.

\section{Relative Capacity, Robin function, and convergence theorems}

Conditions replacing monotone convergence of $v_{j} \rightarrow v$ under which the MongeAmpere measures $\left(d d^{c} v_{j}\right)^{N}$ converge weak-* to $\left(d d^{c} v\right)^{N}$ have been studied by Y. Xing [X]. We say that $v_{j} \rightarrow v$ in capacity on $B$ if, for each $F \subset \subset B$,

$$
\lim _{j \rightarrow+\infty} C\left(\left\{z \in F:\left|v_{j}(z)-v(z)\right|>\delta\right\}\right)=0
$$

for all $\delta>0$. Xing shows that, e.g., if $\left\{v_{j}\right\}$ is a locally uniformly bounded family of psh functions on $B$ and $v_{j} \rightarrow v$ in capacity on $B$ where $v$ is locally bounded, then 
$\left(d d^{c} v_{j}\right)^{N}$ converge weak-* to $\left(d d^{c} v\right)^{N}$. As in the introduction, we use the notation $u_{j}:=U_{E_{j}}^{*}$ and $u:=U_{E}^{*}$ for the relative extremal functions of the Borel sets $E_{j}$ and $E$ relative to the unit ball $B$; and we denote by $C\left(E_{j}\right)$ and $C(E)$ the relative capacities of $E_{j}$ and $E$ relative to $B$. Our first result of this section is the following.

Theorem 1.1. Let $\left\{E_{j}\right\}$ be a sequence of Borel subsets of a given non-pluripolar Borel set $E$ in the unit ball $B$ in $\mathbf{C}^{N}$ with $E \subset \subset B$. The following statements are equivalent:

(i) $\lim _{j \rightarrow \infty} C\left(E_{j}\right)=C(E)$;

(ii) $u_{j} \rightarrow u$ in capacity on $B$ and $\mu_{j}:=\left(d d^{c} u_{j}\right)^{N}$ converge weak-* to $\mu:=$ $\left(d d^{c} u\right)^{N}$

(iii) $u_{j} \rightarrow u$ pointwise on $B$;

(iv) $V_{E_{j}}^{*} \rightarrow V_{E}^{*}$ pointwise on $\mathbf{C}^{N}$.

Proof of Theorem 1.1. We may assume that each $E_{j}$ is non-pluripolar.

I. (i) implies (ii): We proceed with a series of results.

Proposition 1.1. Suppose $\lim _{j \rightarrow \infty} C\left(E_{j}\right)=C(E)$. Then for $k=0,1, \ldots, N$, the sequences

$$
\int_{B} u_{j}\left(d d^{c} u_{j}\right)^{k} \wedge\left(d d^{c} u\right)^{N-k}, \int_{B} u\left(d d^{c} u_{j}\right)^{k} \wedge\left(d d^{c} u\right)^{N-k}
$$

converge to $\int_{B} u\left(d d^{c} u\right)^{N}$ as $j \rightarrow \infty$.

Proof. Recall that $-C\left(E_{j}\right)=\int_{B} u_{j}\left(d d^{c} u_{j}\right)^{N}$ and note that $E_{j} \subset E$ implies $u_{j} \geq u$. Thus, by applying this inequality and repeated integration by parts,

$$
\begin{aligned}
-C\left(E_{j}\right) & =\int_{B} u_{j}\left(d d^{c} u_{j}\right)^{N} \geq \int_{B} u\left(d d^{c} u_{j}\right)^{N} \\
& =\int_{B} u_{j}\left(d d^{c} u\right) \wedge\left(d d^{c} u_{j}\right)^{N-1} \geq \ldots \geq \int_{B} u\left(d d^{c} u\right)^{N}=-C(E) .
\end{aligned}
$$

Since $\lim _{j \rightarrow \infty} C\left(E_{j}\right)=C(E)$, the result follows.

Remark. The integration by parts are justified since $u_{j}(z) \geq u(z) \geq A\left(|z|^{2}-1\right)$ for all $z \in B$ if $A>0$ is sufficiently large (cf., [C], Theorem 3.1.3).

Corollary 1.1. Suppose $\lim _{j \rightarrow \infty} C\left(E_{j}\right)=C(E)$. Then $u_{j} \rightarrow u$ in capacity on $B$.

Proof. Following the proof of Theorem 2 in [X], for $F \subset \subset B$, given $w$ psh in $B$ with $0 \leq w \leq 1$ and given $\delta>0$,

$$
\begin{aligned}
\int_{F \cap\left\{u_{j}-u>\delta\right\}}\left(d d^{c} w\right)^{N} & \leq \frac{1}{\delta} \int_{B}\left(u_{j}-u\right)\left(d d^{c} w\right)^{N} \\
& \leq C(N, \delta)\left(\int_{B}\left(u_{j}-u\right)\left[\left(d d^{c} u_{j}\right)^{N}-\left(d d^{c} u\right)^{N}\right]\right)^{1 / 2^{N}}
\end{aligned}
$$

for some constant $C(N, \delta)$. The result now follows from Proposition 1.1.

Using Theorem 1 (i) of $[\mathrm{X}]$ and the uniform boundedness of $\left\{u_{j}\right\}, u$, we get convergence of the relative equilibrium measures $\mu_{j}$ to $\mu$.

II. (ii) implies (i): If the relative equilibrium measures $\mu_{j}$ converge to $\mu$, then, as in the introduction, if $\phi \in C_{0}^{\infty}(B)$ is nonnegative and equal to one on $\bar{E}$,

$$
\lim _{j \rightarrow \infty} C\left(E_{j}\right)=\lim _{j \rightarrow \infty} \int_{B} \phi\left(d d^{c} u_{j}\right)^{N}=\int_{B} \phi\left(d d^{c} u\right)^{N}=C(E) .
$$


III. (i) implies (iii): We need a lemma about subharmonic functions. Below, $m$ denotes Lebesgue measure in $\mathbf{C}^{N}$ and $B(0, r)$ denotes the ball of radius $r$ centered at the origin.

Lemma 1.1. Fix $0<r<1$ and $0 \leq a<b \leq 1$. There exists $\delta=\delta(r, a, b)>0$ such that for all $v$ subharmonic in $B$ with $0 \leq v \leq 1$ and $v(0) \geq b$,

$$
m(\{z \in B(0, r): v(z)>a\})>\delta .
$$

Proof. For any such $v$, let $E:=\{z \in B(0, r): v(z)>a\}$. Then

$$
\begin{aligned}
b & \leq v(0) \leq \frac{1}{m(B(0, r))} \int_{B(0, r)} v=\frac{\int_{E} v+\int_{B(0, r) \backslash E} v}{m(B(0, r))} \\
& \leq \frac{\int_{E} v+a[m(B(0, r))-m(E)]}{m(B(0, r))} .
\end{aligned}
$$

Hence

$$
(b-a) m(B(0, r)) \leq \int_{E}(v-a) \leq(1-a) m(E)
$$

which implies

$$
m(E) \geq\left(\frac{b-a}{1-a}\right) m(B(0, r))
$$

Now to show that $u_{j} \rightarrow u$ pointwise on $B$, fix a point in $B$ which, for simplicity, we take to be the origin. Let $v_{j}:=u_{j}+1$ and $v:=u+1$. We show $\lim _{j \rightarrow \infty} v_{j}(0)=$ $v(0)$. Since $E_{j} \subset E, v_{j}(0) \geq v(0)$; suppose, for the sake of obtaining a contradiction, that there is a subsequence $\left\{v_{n_{j}}(0)\right\}$ with $v_{n_{j}}(0) \geq b>v(0)$. Fix $a$, $a^{\prime}$ with $v(0)<a^{\prime}<a<b$. By uppersemicontinuity of $v$ at 0 , there exists $r>0$ such that $v(z)<a^{\prime}$ for $z \in B(0, r)$. For this $r$, we apply Lemma 1.1 to conclude that

$$
m\left(\left\{z \in B(0, r): v_{n_{j}}(z)>a\right\}\right)>\delta
$$

for all $j$. But then since Lebesgue measure is dominated by the relative capacity [BT1],

$$
C\left(\left\{z \in B(0, r): v_{n_{j}}(z)-v(z)>a-a^{\prime}\right\}\right) \geq C\left(\left\{z \in B(0, r): v_{n_{j}}(z)>a\right\}\right)>\delta^{\prime}
$$

for all $j$ where $\delta^{\prime}=\delta^{\prime}(\delta, a, b, r)>0$ is independent of $j$. This latter inequality contradicts the convergence of $u_{j}$ to $u$ in capacity on $B$ (Corollary 1.1).

IV. (iii) implies (ii): Since $E_{j} \subset E$ implies $u_{j} \geq u$, we obtain the inequality

$$
u(z) \leq u_{j}(z) \leq v_{j}(z):=\sup _{s \geq j} u_{s}(z) \leq v_{j}^{*}(z) \leq 0
$$

for $z \in B$ and each $j$. We show that $v_{j}^{*} \rightarrow u$ in capacity on $B$; by the above inequality, it will then follow that $u_{j} \rightarrow u$ in capacity on $B$. The argument below may be found in $[\mathrm{Ko}]$, Corollary 1.2.10. Recall that there exists a constant $A>0$ so that for all $z \in B, A\left(|z|^{2}-1\right) \leq u(z)$. Fix $F \subset \subset B$ and choose $A$ sufficiently large so that

$$
A\left(|z|^{2}-1\right) \leq u(z)-1 \text { on } \bar{F} .
$$


Then $\tilde{u}(z):=\max \left[u(z)-1, A\left(|z|^{2}-1\right)\right]$ and $\tilde{v}_{j}(z):=\max \left[v_{j}^{*}(z)-1, A\left(|z|^{2}-1\right)\right]$ are uniformly bounded psh functions in $B$ which agree on a neighborhood of $\partial B$. Furthermore, $\tilde{v}_{j}(z)$ decrease pointwise q.e. on $B$ to $\tilde{u}(z)$ since

$$
u(z)=\limsup _{j \rightarrow \infty} u_{j}(z)=\lim _{j \rightarrow \infty} v_{j}(z)
$$

for $z \in B$. Using Theorem 3.4 of [BT1], we conclude that for any $\delta>0$,

$$
\lim _{j \rightarrow \infty} C\left(\left\{z \in B: \tilde{v}_{j}(z)>\tilde{u}(z)+\delta\right\}\right)=0 .
$$

Since $\left\{z \in F: v_{j}^{*}(z)>u(z)+\delta\right\} \subset\left\{z \in B: \tilde{v}_{j}(z)>\tilde{u}(z)+\delta\right\}$, we conclude that $v_{j}^{*} \rightarrow u$ in capacity on $B$. As before, the convergence of the relative equilibrium measures $\mu_{j} \rightarrow \mu$ follows from Theorem 1 (i) of [X] and the uniform boundedness of $\left\{u_{j}\right\}$ and $u$.

V. (i) implies (iv): We first show that $V_{E_{j}}^{*}$ converges pointwise to 0 q.e. on $E$. Without loss of generality, we may assume that there is an $r<1$ such that the ball $B(0, r)$ of radius $r$ centered at the origin contains $E$ (and hence each $E_{j}$ ). By hypothesis, there exists $j_{0}$ such that $C\left(E_{j}\right)>C(E) / 2$ for $j>j_{0}$. Using the capacity comparison theorem of Alexander and Taylor (Theorem 2.1, eqn. (2.7) in [AT]; see also Theorem $3.2[\mathrm{AT}]$ ), there exists a constant $A$ depending only on $r$ such that

$$
\sup _{z \in B} V_{E_{j}}^{*}(z) \leq \frac{A}{C\left(E_{j}\right)}
$$

Hence,

$$
\sup _{z \in B} V_{E_{j}}^{*}(z)<\frac{2 A}{C(E)} \text { for all } j>j_{0} .
$$

Thus we get the trivial estimate

$$
V_{E_{j}}^{*}(z) \leq \frac{2 A}{C(E)}\left(u_{j}(z)+1\right) \text { for all } z \in B
$$

for $j>j_{0}$ (cf., Proposition 5.3.3 [K]). In particular, for $z \in E$, we have

$$
0 \leq V_{E_{j}}^{*}(z) \leq \frac{2 A}{C(E)}\left(u_{j}(z)+1\right)
$$

since $u_{j}$ converges pointwise to $u$ on $B$ and $u=-1$ q.e. on $E$, we conclude that $V_{E_{j}}^{*}$ converges pointwise to 0 q.e. on $E$.

We now show

$$
\lim _{j \rightarrow \infty} V_{E_{j}}^{*}(z)=V_{E}^{*}(z) \text { pointwise on all of } \mathbf{C}^{N} .
$$

Let $w_{s}(z):=\sup _{j \geq s} V_{E_{j}}^{*}(z)$. Note that $w_{s}^{*} \in L$ for sufficiently large $s$ since, by $(1.2)$,

$$
\sup _{B} w_{s} \leq \frac{2 A}{C(E)} \text { for all } s>j_{0}
$$

Indeed,

$$
V_{E}^{*}(z) \leq V_{E_{s}}^{*}(z) \leq w_{s}(z) \leq \frac{2 A}{C(E)}+\log ^{+}|z|
$$


so that $w_{s}^{*} \in L_{+}$. Note that the sequence $\left\{w_{s}\right\}$ is decreasing; since $V_{E_{j}}^{*}$ converges pointwise to 0 q.e. on $E$, we have that

$$
w(z):=\lim _{s \rightarrow \infty} w_{s}(z)=\limsup _{j \rightarrow \infty} V_{E_{j}}^{*}(z)=0 \text { q.e. on } E .
$$

Thus the usc regularization $w^{*}(z)=0$ q.e. on $E$; and, by (1.4), $w^{*} \in L_{+}$. From Lemma 6.5 of [BT2], we conclude that

$$
w^{*}(z) \leq V_{E}^{*}(z) \text { pointwise on all of } \mathbf{C}^{N}
$$

On the other hand, since $E_{j} \subset E$, we have $V_{E}^{*} \leq V_{E_{j}}^{*}$ so that for all $j$ and for all $z \in \mathbf{C}^{N}$,

Thus, for all $z \in \mathbf{C}^{N}$,

$$
w^{*}(z) \leq V_{E}^{*}(z) \leq V_{E_{j}}^{*}(z)
$$

$$
w(z) \leq w^{*}(z) \leq V_{E}^{*}(z) \leq \liminf _{j \rightarrow \infty} V_{E_{j}}^{*}(z) \leq \limsup _{j \rightarrow \infty} V_{E_{j}}^{*}(z)=w(z)
$$

Thus equality holds throughout; in particular, $\lim _{j \rightarrow \infty} V_{E_{j}}^{*}(z)$ exists and equals $V_{E}^{*}(z)$; i.e., (1.3) is proved.

VI. (iv) implies (iii): From Proposition 5.3 .3 of $[\mathrm{K}]$, for $z \in B$,

$$
V_{E_{j}}^{*}(z) \geq\left(\inf _{\partial B} V_{E_{j}}^{*}\right)\left[u_{j}(z)+1\right] \geq 0 .
$$

Since $V_{E_{j}}^{*}$ converges pointwise q.e. to 0 on $E$, we conclude that $u_{j}$ converges pointwise q.e. to -1 on $E$. Next, we recall the inequality

$$
u(z) \leq u_{j}(z) \leq v_{j}(z):=\sup _{s \geq j} u_{s}(z)
$$

for $z \in B$ and each $j$ (since $E_{j} \subset E$ ). Thus $\left\{v_{j}\right\}$ is a decreasing sequence of functions which converges to -1 q.e. on $E$; i.e.,

$$
v(z):=\lim _{j \rightarrow \infty} v_{j}(z)=\limsup _{j \rightarrow \infty} u_{j}(z)
$$

equals -1 q.e. on $E$ from which we conclude that $v^{*}(z) \leq u(z)$ on $B$. Thus, combining with (1.5), we have

$$
v(z) \leq v^{*}(z) \leq u(z) \leq \liminf _{j \rightarrow \infty} u_{j}(z) \leq \limsup _{j \rightarrow \infty} u_{j}(z)=v(z)
$$

for all $z \in B$ so that equality holds throughout; i.e., $\lim _{j \rightarrow \infty} u_{j}(z)$ exists and equals $u(z)=v(z)=v^{*}(z)$.

If $E$ is compact and regular, the convergence of the extremal functions is stronger; the extra ingredient in the proof of the result below is Hartogs' lemma (Theorem $2.6 .4[\mathrm{~K}])$.

Theorem 1.2. Let $\left\{K_{j}\right\}$ be a sequence of compact subsets of a given compact set $K$ in the unit ball $B$ in $\mathbf{C}^{N}$; let $u_{j}:=U_{K_{j}}^{*}$ and $u:=U_{K}^{*}$. Suppose $K$ is regular. Then the following statements are equivalent:

(i) $\lim _{j \rightarrow \infty} C\left(K_{j}\right)=C(K)$;

(ii) $u_{j} \rightarrow u$ in capacity on $B$ and $\mu_{j}:=\left(d d^{c} u_{j}\right)^{N}$ converge weak-* to $\mu:=$ $\left(d d^{c} u\right)^{N}$

(iii) $u_{j} \rightarrow u$ uniformly on $B$;

(iv) $V_{K_{j}}^{*} \rightarrow V_{K}^{*}$ uniformly on $\mathbf{C}^{N}$ and the global equilibrium measures $\left(d d^{c} V_{K_{j}}^{*}\right)^{N}$ converge weak-* ${ }^{*}$ to $\left(d d^{c} V_{K}\right)^{N}$. 
We briefly indicate how one obtains the (global!) uniform convergence in (iv) from the pointwise convergence of $V_{K_{j}}^{*} \rightarrow V_{K}^{*}$. Using the uniform boundedness of $\left\{V_{K_{j}}^{*}\right\}$ on $B$ for large $j$ (cf., (1.2)) together with Hartogs' lemma and regularity of $K$ yields uniform convergence of $V_{K_{j}}^{*} \rightarrow 0$ on $K$. Thus, given $\epsilon>0$, we have $V_{K_{j}}^{*} \leq \epsilon$ on $K$ for sufficiently large $j$; thus $V_{K}^{*}-\epsilon \leq V_{K_{j}}^{*}-\epsilon \leq V_{K}^{*}$ on $\mathbf{C}^{N}$ for sufficiently large $j$. The uniform convergence in (iii) is obtained in a similar manner.

A consequence of (iv) will be used in the next section (cf., Proposition 2.2; see also Corollary 3.1).

Corollary 1.2. Let $K \subset B$ be a regular, compact set. Given $\epsilon>0$, there exists $\delta>0$ such that for any compact subset $E \subset K$ satisfying $C(E)>C(K)-\delta$, we have $V_{E}^{*}(z) \leq \epsilon$ for $z \in K$.

We next recall the notion of the Robin function associated to a function $u \in L$. For such a function, we define

$$
\rho_{u}(z)=\limsup _{\substack{|\lambda| \rightarrow+\infty \\ \lambda \in \mathbf{C}}}\left\{u(\lambda z)-\log ^{+}|\lambda z|\right\}
$$

and

$$
\bar{\rho}_{u}(z)=\limsup _{\substack{|\lambda| \rightarrow+\infty \\ \lambda \in \mathbf{C}}}\{u(\lambda z)-\log |\lambda|\} .
$$

Following the convention used by Bedford and Taylor [BT2], we consider $\rho_{u}$ to be defined on $\mathbf{P}^{N-1}$ (complex projective $(N-1)$-space) and refer to it as the Robin function of $u$. We let $[z]$ denote the point in $\mathbf{P}^{N-1}$ determined by $z \in \mathbf{C}^{N}-\{0\}$, and we use the notation $\rho_{u}([z])$ for the value of the Robin function at this point. Clearly

$$
\rho_{u}([z])=\bar{\rho}_{u}(z)-\log |z| \quad \text { for } \quad z \in \mathbf{C}^{N}-\{0\} .
$$

For $E \subset \mathbf{C}^{N}$, the Robin function of $E$, denoted by $\rho_{E}$, is defined to be the Robin function of $V_{E}^{*}$. We recall the following result (cf., [Bl2]).

Proposition 1.2. (i) Let $u \in L$. Then $\bar{\rho}_{u}$ is psh on $\mathbf{C}^{N}$.

(ii) Let $u \in L$. Then $\rho_{u}$ is uppersemicontinuous on $\mathbf{P}^{N-1}$.

(iii) Let $u, v \in L$. Suppose that $\rho_{u}=\rho_{v}$ almost everywhere (a.e.) on $\mathbf{P}^{N-1}$. Then $\rho_{u}=\rho_{v}$ at all points of $\mathbf{P}^{N-1}$.

(iv) For $K$ compact and regular, $\rho_{K}$ is continuous on $\mathbf{P}^{N-1}$.

Proposition 1.3. Under the hypothesis of Theorem 1.1, the following are equivalent:

(a) $V_{E_{j}}^{*} \rightarrow V_{E}^{*}$ pointwise on $\mathbf{C}^{N}$;

(b) $\rho_{E_{j}} \rightarrow \rho_{E}$ pointwise on $\mathbf{P}^{N-1}$.

Proof. (a) implies (b): Using the notation from part $\mathbf{V}$ of the proof of Theorem 1.1, we recall that $\left\{w_{s}\right\}$ is a sequence of functions in $L_{+}$which decreases pointwise to $V_{E}^{*}$ on $\mathbf{C}^{N}$. From the discussion on p. 136 of [BT2], it follows that the corresponding Robin functions $\rho_{w_{s}}$ decrease pointwise to $\rho_{E}$. But $w_{s} \geq V_{E_{s}}^{*} \geq V_{E}^{*}$ for all $s$; thus the Robin functions $\rho_{E_{j}}$ also converge pointwise to $\rho_{E}$.

(b) implies (a): The hypothesis implies that $\bar{\rho}_{E_{j}} \rightarrow \bar{\rho}_{E}$ pointwise on $\mathbf{C}^{N}$. Since $\bar{\rho}_{E_{j}}, \bar{\rho}_{E} \in L$, by Lemma 3.2 of [Bl1], $\left\{\bar{\rho}_{E_{j}}\right\}$ is locally uniformly bounded above on 
$\mathbf{C}^{N}$. Hence $\left\{\rho_{E_{j}}\right\}$ is uniformly bounded above on $\mathbf{P}^{N-1}$. We now proceed with several steps.

Step 1. $\lim _{j \rightarrow \infty} \int_{\mathbf{C}^{N}} V_{E_{j}}^{*}\left(d d^{c} V_{E}^{*}\right)^{N}=0$.

Proof. We first set up some notation, following [BT2]. We consider $\mathbf{C}^{N} \subset \mathbf{P}^{N}$, where $\left(z_{1}, \ldots, z_{N}\right) \in \mathbf{C}^{N}$ correspond to homogeneous coordinates $\left[1, z_{1}, \ldots, z_{N}\right] \in$ $\mathbf{P}^{N}$; i.e., $\left(z_{1}, \ldots, z_{N}\right)$ give local coordinates for $U_{0}:=\left\{\left[z_{0}, z_{1}, \ldots, z_{N}\right] \in \mathbf{P}^{N}: z_{0} \neq 0\right\}$. We work in the coordinate chart $U_{1}:=\left\{\left[z_{0}, z_{1}, \ldots, z_{N}\right] \in \mathbf{P}^{N}: z_{1} \neq 0\right\}$ with local coordinates $\left(t^{\prime}, t\right):=\left(t^{\prime}, t_{2}, \ldots, t_{N}\right)$ of $\mathbf{C}^{N}$ where $z_{1}=1 / t^{\prime}, z_{j}=t_{j} / t^{\prime}, j=2, \ldots, N$. Then $t^{\prime}=0$ corresponds to the hyperplane at infinity intersected with $U_{1}$ and for a function $u$ in the class $L_{+}$, the function

$$
\tilde{u}\left(t^{\prime}, t\right):=u\left(1 / t^{\prime}, t / t^{\prime}\right)+\log \left|t^{\prime}\right|
$$

belongs to $L_{+}$in the $\left(t^{\prime}, t\right)$ coordinates. It follows that

$$
\rho_{u}(t)=\tilde{u}(0, t)-\frac{1}{2} \log \left(1+|t|^{2}\right)
$$

and we define

$$
\Omega(u):=d d^{c} \tilde{u}(0, t)=d d^{c} \rho_{u}+d d^{c} \frac{1}{2} \log \left(1+|t|^{2}\right) .
$$

Since $V_{E_{j}}^{*}, V_{E}^{*} \in L_{+}$, by the equation below (6.6) in [BT2],

$$
\int_{\mathbf{P}^{N-1}} \Omega\left(V_{E}^{*}\right)^{N-1-s} \wedge \Omega\left(V_{E_{j}}^{*}\right)^{s}=(2 \pi)^{N-1} \text { for } s=0,1, \ldots, N-1 .
$$

Now using Theorem 6.1 in [BT2],

$$
\begin{aligned}
0 \leq \int_{\mathbf{C}^{N}} & V_{E_{j}}^{*}\left(d d^{c} V_{E}\right)^{N} \leq \int_{\mathbf{C}^{N}} V_{E}\left(d d^{c} V_{E_{j}}^{*}\right)^{N} \\
& +\sum_{s=0}^{N-1} 2 \pi \int_{\mathbf{P}^{N-1}}\left(\rho_{E_{j}}-\rho_{E}\right) \Omega\left(V_{E}^{*}\right)^{N-1-s} \wedge \Omega\left(V_{E_{j}}^{*}\right)^{s}
\end{aligned}
$$

for $j=1,2, \ldots$. Since $V_{E}^{*}=0$ q.e. on $E \supset E_{j}$,

$$
\int_{\mathbf{C}^{N}} V_{E}\left(d d^{c} V_{E_{j}}^{*}\right)^{N}=0
$$

and we have

$$
0 \leq \int_{\mathbf{C}^{N}} V_{E_{j}}^{*}\left(d d^{c} V_{E}\right)^{N} \leq \sum_{s=0}^{N-1} 2 \pi \int_{\mathbf{P}^{N-1}}\left(\rho_{E_{j}}-\rho_{E}\right) \Omega\left(V_{E}^{*}\right)^{N-1-s} \wedge \Omega\left(V_{E_{j}}^{*}\right)^{s} .
$$

We claim that for each $s=0,1, \ldots, N-1$,

$$
\limsup _{j \rightarrow \infty} \int_{\mathbf{P}^{N-1}}\left(\rho_{E_{j}}-\rho_{E}\right) \Omega\left(V_{E}^{*}\right)^{N-1-s} \wedge \Omega\left(V_{E_{j}}^{*}\right)^{s}=0 .
$$

Assuming (1.7), we then have

$$
\limsup _{j \rightarrow \infty} \int_{\mathbf{C}^{N}} V_{E_{j}}^{*}\left(d d^{c} V_{E}^{*}\right)^{N}=0
$$

Since $V_{E_{j}}^{*} \geq 0$, the conclusion of Step 1 follows from the obvious inequalities

$$
0 \leq \liminf _{j \rightarrow \infty} \int_{\mathbf{C}^{N}} V_{E_{j}}^{*}\left(d d^{c} V_{E}^{*}\right)^{N} \leq \limsup _{j \rightarrow \infty} \int_{\mathbf{C}^{N}} V_{E_{j}}^{*}\left(d d^{c} V_{E}^{*}\right)^{N}=0 .
$$


For simplicity of notation, let $v_{j}(t):=\tilde{V}_{E_{j}}^{*}(0, t)$ and $v(t):=\tilde{V}_{E}^{*}(0, t)$; we work in $\mathbf{C}^{N-1}$. Note that $v_{j} \rightarrow v$ pointwise on $\mathbf{C}^{N-1}$ and, from the uniform boundedness of the Robin functions, for each fixed $R>0$, the functions $v_{j}, v$ are uniformly bounded on $B(0, R) \subset \mathbf{C}^{N-1}$ (below by 0 ; above by $M+\log R$ for a fixed $M=$ $M(R)=o(\log R))$. From (1.6), the measures $\left(d d^{c} v\right)^{N-1-s} \wedge\left(d d^{c} v_{j}\right)^{s}$ each have finite total mass. Fix $\epsilon>0$ and choose $R=R(\epsilon)$ so that

$$
\int_{\mathbf{C}^{N-1} \backslash B(0, R)}\left(d d^{c} v\right)^{N-1-s} \wedge\left(d d^{c} v_{j}\right)^{s}<\epsilon
$$

for $j>J(\epsilon)$. This can be done from the uniform boundedness of the Robin functions $\left\{\rho_{E_{j}}\right\}$ on $\mathbf{P}^{N-1}$ and Proposition 3.1 of [BT2]:

$$
\int_{\mathbf{C}^{N-1} \backslash B(0, R)}\left(d d^{c} \tilde{V}_{E}^{*}(0, t)\right)^{N-1-s} \wedge\left(d d^{c} \tilde{V}_{E_{j}}^{*}(0, t)\right)^{s}=o\left(\frac{1}{\log R}\right)
$$

uniformly in $j$.

Note that since

$$
\begin{aligned}
\left(\rho_{E_{j}}\right. & \left.-\rho_{E}\right) \Omega\left(V_{E}^{*}\right)^{N-1-s} \wedge \Omega\left(V_{E_{j}}^{*}\right)^{s} \\
& =\left[\tilde{V}_{E_{j}}^{*}(0, t)-\tilde{V}_{E}^{*}(0, t)\right]\left(d d^{c} \tilde{V}_{E}^{*}(0, t)\right)^{N-1-s} \wedge\left(d d^{c} \tilde{V}_{E_{j}}^{*}(0, t)\right)^{s},
\end{aligned}
$$

our goal is to show that for each $s=0, \ldots, N-1$,

$$
\limsup _{j \rightarrow \infty} \int_{\mathbf{C}^{N-1}}\left(v_{j}-v\right)\left(d d^{c} v\right)^{N-1-s} \wedge\left(d d^{c} v_{j}\right)^{s}=0 .
$$

From the uniform boundedness of $v_{j}-v$ and (1.8), it suffices to show

$$
\limsup _{j \rightarrow \infty} \int_{B(0, R)}\left(v_{j}-v\right)\left(d d^{c} v\right)^{N-1-s} \wedge\left(d d^{c} v_{j}\right)^{s}=0 .
$$

Fix $R^{\prime}>R$. From the uniform boundedness of the functions $v_{j}, v$ on $B\left(0, R^{\prime}\right)$ and pointwise convergence of $v_{j} \rightarrow v$, by use of Lemma 1.1 and the argument afterwards, it follows that $v_{j} \rightarrow v$ in capacity (relative to $B\left(0, R^{\prime}\right)$ ) on $B\left(0, R^{\prime}\right)$. Now (1.9) follows from the proof of Theorem 1 (ii) of [X].

Step 2. $V_{E_{j}}^{*}$ converges pointwise q.e. to 0 on $E$.

Proof. Let $E^{\prime}:=\left\{z \in E: V_{E}^{*}(z)=0\right\}$. Then $E \backslash E^{\prime}$ is pluripolar; we show that $V_{E_{j}}^{*}$ converges pointwise to 0 on $E^{\prime}$. Suppose not; i.e., suppose there is a point $z_{0} \in E^{\prime}$ with

$$
\limsup _{j \rightarrow \infty} V_{E_{j}}^{*}\left(z_{0}\right)>0 \text {. }
$$

We take a subsequence $J_{1}$ of the positive integers such that

$$
\lim _{j \rightarrow \infty, j \in J_{1}} V_{E_{j}}^{*}\left(z_{0}\right)>0 .
$$

Using Step 1, the sequence $\left\{V_{E_{j}}^{*}\right\}_{j \in J_{1}}$ converges to 0 in $\mu_{E}$-measure. Thus we can take a further subsequence $J_{2} \subset J_{1}$ with $\left\{V_{E_{j}}^{*}\right\}_{j \in J_{2}}$ converging to $0 \mu_{E}$-almost everywhere on $E$.

Define $v(z):=\left[\lim \sup _{j \rightarrow \infty}, j \in J_{2} V_{E_{j}}^{*}(z)\right]^{*}$. Then $v=0$ q.e.- $\mu_{E}$; i.e., there is a (possibly empty) pluripolar set $Z$ with $v=0$ a.e. $\mu_{E}$ on $E \backslash Z$. Since $E$ is non-pluripolar, $\mu_{E}$ is a determining measure and $V_{\mu_{E}}=V_{E}^{*}$ (see [Bl1] for the 
terminology). Using Proposition 3.4 [Bl1], it follows that $v \leq V_{\mu_{E}}=V_{E}^{*}$. In particular, at $z_{0}$,

$$
V_{E}^{*}\left(z_{0}\right) \geq v\left(z_{0}\right) \geq \lim _{j \rightarrow \infty, j \in J_{2}} V_{E_{j}}^{*}\left(z_{0}\right)=\lim _{j \rightarrow \infty, j \in J_{1}} V_{E_{j}}^{*}\left(z_{0}\right)>0,
$$

a contradiction.

Step 3. $V_{E_{j}}^{*} \rightarrow V_{E}^{*}$ pointwise on $\mathbf{C}^{N}$.

Proof. As in the proof of step $\mathbf{V}$ of Theorem 1.1, we let $w(z):=\lim \sup _{j \rightarrow \infty} V_{E_{j}}^{*}(z)$. From Step $2, w^{*}(z)=0$ q.e. on $E$; and by Lemma 3.2 [Bl1], $w^{*} \in L$. Using [BT2], Lemma 6.5 , we conclude that $w^{*} \leq V_{E}^{*}$ on $\mathbf{C}^{N}$. Since $V_{E}^{*} \leq V_{E_{j}}^{*}$, we obtain the string of inequalities

$$
w^{*} \leq V_{E}^{*} \leq \liminf _{j \rightarrow \infty} V_{E_{j}}^{*} \leq \limsup _{j \rightarrow \infty} V_{E_{j}}^{*}=w \leq w^{*}
$$

valid on all of $\mathbf{C}^{N}$. Thus equality holds throughout and the result follows.

\section{Sufficient MAss-Density CONDITIONS FOR Bernstein-Markov}

We return to the situation described in the introduction, letting $\mu$ be a finite positive Borel measure with compact support $K:=S(\mu)$ in $\mathbf{C}^{N}$. For simplicity, we assume $S(\mu) \subset B$. Let $B(z, r)$ denote the closed ball of radius $r$ centered at $z$. We can now state the result in [Bl1] (Theorem 4.1).

Theorem 2.1. Suppose there exists a positive constant $T$ such that

$$
C\left(\left\{z: \liminf _{r \rightarrow 0^{+}} \frac{\mu(B(z, r))}{r^{T}} \geq 1\right\}\right)=C(S(\mu)) .
$$

If $K:=S(\mu)$ is regular, then $(K, \mu)$ satisfy (0.1).

Roughly speaking, condition (2.1) says that the measure is sufficiently dense on a set of full relative capacity; the example of the discrete measure $\mu$ described in the introduction shows that in some sense, such a set can be very "sparse". We give a slightly stronger result, showing that one only needs a type of denseness in the mean.

Theorem 2.2. Suppose there exists a positive constant $T$ such that

$$
\lim _{r \rightarrow 0^{+}} C\left(\left\{z: \mu(B(z, r)) \geq r^{T}\right\}\right)=C(S(\mu)) .
$$

If $K:=S(\mu)$ is regular, then $(K, \mu)$ satisfy (0.1).

Remark. Theorem 2.2 is a direct several-variable analogue of Theorem 4.2.3 [ST].

If a measure satisfies the mass-density criterion (2.1) in Theorem 2.1, then clearly it satisfies criterion (2.2) of Theorem 2.2; since the proofs of these theorems are similar, we outline the reasoning for both and give the details of the proof of Theorem 2.2 below. The key ingredient in each proof is an approximation result for a regular compact set; Proposition 2.1 is used for Theorem 2.1 and Proposition 2.2 is used for Theorem 2.2.

Proposition 2.1 (Proposition 2.1 [Bl1]). Let $K$ be a regular compact set in $\mathbf{C}^{N}$ and let $\left\{E_{j}\right\}$ be an increasing sequence of Borel subsets of $K$ with $\lim _{j \rightarrow \infty} C\left(E_{j}\right)=$ $C(K)$. Then there exists an increasing family of compact sets $\left\{L_{j}\right\}$ with $L_{j} \subset E_{j}$ such that, given $\epsilon>0$, there exists $J=J(\epsilon)$ so that for $j>J, V_{L_{j}}^{*} \leq V_{K}+\epsilon$. 
Proposition 2.2. Let $K$ be a regular compact set in $\mathbf{C}^{N}$ and let $\left\{K_{j}\right\}$ be a sequence of compact subsets of $K$ with $\lim _{j \rightarrow \infty} C\left(K_{j}\right)=C(K)$. Then given $\epsilon>0$, there exists $J=J(\epsilon)$ so that for $j>J, V_{K_{j}}^{*} \leq V_{K}+\epsilon$.

Remark. Note that Proposition 2.2 is equivalent to Corollary 1.2 of the previous section; we state this version to draw the analogy with Proposition 2.1. It also follows from Proposition 1.3 that in Proposition 2.2, the hypothesis that $\lim _{j \rightarrow \infty} C\left(K_{j}\right)=C(K)$ may be replaced by $\rho_{K_{j}} \rightarrow \rho_{K}$ pointwise (equivalently, uniformly) on $\mathbf{P}^{N-1}$. Thus, the mass-density condition (2.2) can also be reformulated in terms of the Robin function.

We outline the main ideas involved in the proofs of both theorems and then we give the precise proof of Theorem 2.2; this also proves Theorem 2.1 (for details of the original proof of Theorem 2.1, see [Bl1]).

Given $\epsilon>0$, we choose $\delta=\delta(\epsilon)$ sufficiently small so that if we set $K_{\delta}:=\{z \in$ $\left.\mathbf{C}^{N}: d(z, K) \leq \delta\right\}$, then:

1. $V_{K}(z) \leq \epsilon$ for $z \in K_{\delta}$;

thus for a polynomial $p$ of degree $d$, the Bernstein-Walsh inequality (cf., $[\mathrm{K}]$ ) is valid:

$$
\|p\|_{K_{\delta}} \leq\|p\|_{K} e^{\epsilon d}
$$

and we find a compact subset $E$ of $K$ - chosen appropriately using the massdensity condition - with

2. $V_{E}^{*} \leq V_{K}+\epsilon$.

In Theorem 2.1, $E$ will be chosen as $L_{j}$ (Proposition 2.1) for $j$ sufficiently large, while in Theorem 2.2, $E$ will be chosen as $K_{j}$ (Proposition 2.2) for $j$ sufficiently large.

In the next step, we fix a polynomial $p$ of degree $d$ and $w \in E$ with $|p(w)|=|| p||_{E}$ and we show that

$$
|p(z)| \geq \frac{1}{2}|| p \|_{E} \text { if }|z-w|<\frac{\delta}{4} e^{-2 d \epsilon} .
$$

We then take a closed ball $B\left(w, r_{d}\right)$ centered at $w$ with radius $r_{d}$ sufficiently small so that, together with the appropriate mass-density condition, the inequality

$$
\|p\|_{L^{2}(\mu)}^{2} \geq \int_{B\left(w, r_{d}\right)}|p|^{2} d \mu \geq\left[\min _{z \in B\left(w, r_{d}\right)}|p(z)|\right]^{2} \mu\left(B\left(w, r_{d}\right)\right) \geq \frac{1}{4}\|p\|_{E}^{2} \mu\left(B\left(w, r_{d}\right)\right)
$$

yields (0.1).

Proof of Theorem 2.2. We begin as above: given $\epsilon>0$, choose $\delta=\delta(\epsilon)$ sufficiently small so that $V_{K}(z) \leq \epsilon$ for $z \in K_{\delta}$. Then for any polynomial $p$ of degree $d$, we have the Bernstein-Walsh estimate

$$
\|p\|_{K_{\delta}} \leq\|p\|_{K} e^{\epsilon d}
$$

By taking $\delta=\delta(\epsilon)$ smaller, if necessary, for any compact subset $E$ of $K$ with $C(E)>C(K)-\delta$ we have $V_{E}^{*} \leq V_{K}+\epsilon$ from Corollary 1.2. Then for a polynomial $p$ of degree $d$, we have the Bernstein-Walsh estimate

$$
\|p\|_{K} \leq\|p\|_{E} e^{\epsilon d}
$$


Fixing $p$ of degree $d$ and choosing $w \in E$ with $|p(w)|=|| p \|_{E}$, we show that

$$
|p(z)| \geq \frac{1}{2}|| p \|_{E} \text { if }|z-w|<\frac{\delta}{4} e^{-2 d \epsilon} .
$$

We follow the argument in [Bl1]. For $z \neq w$, let $e=\left(e_{1}, \ldots, e_{N}\right):=\frac{z-w}{|z-w|}$ and let

$$
U(t):=p\left(w_{1}+e_{1} t, \ldots, w_{N}+e_{N} t\right)
$$

note this is a polynomial of the single complex variable $t$. Then

$$
p(z)-p(w)=U(|z-w|)-U(0)=\int_{0}^{|z-w|} U^{\prime}(\zeta) d \zeta
$$

so that, since $|z-w|<\frac{\delta}{2}$,

$$
|p(z)-p(w)| \leq|z-w||| U^{\prime} \|_{|t| \leq \frac{\delta}{2}} .
$$

Now for $|t| \leq \delta$, the point $\left(w_{1}+e_{1} t, \ldots, w_{N}+e_{N} t\right)$ lies in $K_{\delta}$ so from (2.3) and (2.5),

$$
\|U\|_{|t| \leq \delta} \leq\|p\|_{E} e^{2 \epsilon d}
$$

Cauchy's inequality applied to $U$ yields $\left\|U^{\prime}\right\|_{|t| \leq \frac{\delta}{2}} \leq \frac{2}{\delta}\|U\|_{|t| \leq \delta}$ and putting this together with (2.6) and the assumption that $|z-w|<\frac{\delta}{4} e^{-2 d \epsilon}$ gives (2.4).

We will take such a set $E$ determined from the mass-density condition (2.2). First, using (2.2), take $T>0$ so that

$$
\lim _{r \rightarrow 0^{+}} C\left(\left\{z: \mu(B(z, r)) \geq r^{T}\right\}\right)=C(K) .
$$

With $\epsilon, \delta$ as above, for $d \geq d(\delta)$ there exist $E_{d} \subset K$ compact as in Corollary 1.2, i.e., with $C\left(E_{d}\right)>C(K)-\delta$ and $V_{E_{d}}^{*}(z) \leq \epsilon$ for $z \in K$, and such that, setting

$$
\begin{gathered}
r_{d}:=e^{-3 d \epsilon}, \\
\mu\left(B\left(x, r_{d}\right)\right) \geq r_{d}^{T}=e^{-3 d T \epsilon} \text { if } x \in E_{d} .
\end{gathered}
$$

We can assume $d$ is sufficiently large so that $r_{d}<\frac{\delta}{4} e^{-2 d \epsilon}$. Then taking $p$ of degree $d$ and $w \in E_{d}$ with $|p(w)|=\|p\|_{E_{d}}$, using (2.4) we obtain

$$
\begin{aligned}
\|p\|_{L^{2}(\mu)}^{2} \geq \int_{B\left(w, r_{d}\right)}|p|^{2} d \mu \geq\left(\min _{z \in B\left(w, r_{d}\right)}|p(z)|^{2}\right) \mu\left(B\left(w, r_{d}\right)\right) \geq \frac{1}{4}\|p\|_{E_{d}}^{2} e^{-3 d T \epsilon} \\
\geq \frac{1}{4}\|p\|_{K}^{2} e^{-3 d T \epsilon-2 d \epsilon}(\text { from }(2.5)) \\
=\frac{1}{4}\|p\|_{K}^{2} e^{-d \epsilon(2+3 T)} \text { for } d \geq d(\delta)
\end{aligned}
$$

which yields the result. 


\section{FinAl REMARKS AND OPEN QUESTIONS}

A compactly supported measure $\mu$ in the complex plane is said to have regular asymptotic behavior, or simply $\mu \in \mathbf{R e g}$, if

$$
\lim _{n \rightarrow \infty} \frac{1}{n} \log \left|p_{n}(z)\right|=V_{K}(z)
$$

for all $z$ outside the convex hull of $K=S(\mu)$, where $\left\{p_{n}\right\}$ are the orthonormal polynomials for $\mu$. In [ST], a sufficient mass-density condition implying that $\mu \in$ Reg is given (Theorem 4.2.1 [ST]); no assumption of regularity of $S(\mu)$ is made. In the proof, use is made of a deep result of Ancona [A], which states that given $K \subset \mathbf{C}$ compact and non-polar, and given $\epsilon>0$, there exists $K^{\prime} \subset K$ compact which is regular and for which $c\left(K^{\prime}\right)>c(K)-\epsilon$. The capacity $c$ here is the socalled logarithmic capacity: $c(K)=\exp [-\gamma(K)]$ where

$$
\gamma(K):=\lim _{|z| \rightarrow+\infty}\left[V_{K}^{*}(z)-\log |z|\right] .
$$

The difficult part of Ancona's theorem is the weaker statement: given $K \subset \mathbf{C}$ compact and non-polar, there exists $K^{\prime} \subset K$ compact which is regular. These results are true in greater generality; e.g., for Newtonian capacities in $\mathbf{R}^{N}, N>2$ $[\mathrm{A}]$.

Problem 1. Is there an Ancona theorem for the relative capacity of compact subsets of the unit ball in $\mathbf{C}^{N}$ for $N>1$ ?

If so, then a generalization to several variables of Theorem 4.2.1 [ST] would follow (see Remark 4.4 [Bl1]). As a possible aid in this direction, we remark that a version of Corollary 1.2 is valid for a regular Borel set.

Corollary 3.1. Let $E$ be a regular Borel subset of B. Given $\epsilon>0$ and $X \subset E$ compact, there exists $\delta=\delta(\epsilon, X)>0$ such that for any $F \subset E$ compact with $C(F)>C(E)-\delta$, we have $V_{F}^{*}(z) \leq \epsilon$ for $z \in X$.

Proof. It suffices to show that if we take any sequence $\left\{E_{j}\right\}$ of compact subsets of $E$ such that

$$
\lim _{j \rightarrow \infty} C\left(E_{j}\right)=C(E)
$$

then $\left\{V_{E_{j}}^{*}\right\}$ converges uniformly to 0 on $X$. By assumption, $V_{E}^{*}=0$ on $E$ so that $V_{E}=V_{E}^{*}$ on $\mathbf{C}^{N}$. Thus for $\epsilon>0$, the sub-level sets

$$
\Theta_{\epsilon / 2}:=\left\{z \in \mathbf{C}^{N}: V_{E}(z)<\epsilon / 2\right\}
$$

are open neighborhoods of $E$. By Theorem 1.1 (iv), $V_{E_{j}}^{*} \rightarrow V_{E}^{*}$ pointwise on $\mathbf{C}^{N}$; thus $\lim \sup _{j \rightarrow \infty} V_{E_{j}}^{*}(z) \leq \epsilon / 2$ for $z \in \Theta_{\epsilon / 2}$. By Hartogs' lemma, given $X \subset E$ compact and $\epsilon>0$, we have $V_{E_{j}}^{*} \leq \epsilon$ on $X$ for $j>j_{0}(\epsilon, X)$.

If one starts with a non-pluripolar compact set $K \subset B \subset \mathbf{C}^{N}$, the set of "regular" points

$$
E:=\left\{z \in K: V_{K}^{*}(z)=0\right\}=\left\{z \in K: U_{K}^{*}(z)=-1\right\}
$$

is a regular Borel set (but not necessarily compact).

Regarding the mass-density condition in Theorem 2.2 , the one-variable counterpart to Theorem 2.2 in [ST] is Theorem 4.2.3; essentially this is best possible for the case $S(\mu)=[0,1]$ (Theorems 4.2.6 and 4.2.7 [ST]). 
Theorem 4.2.8 [ST] gives a necessary condition satisfied by a measure $\mu$ with $S(\mu)=[0,1]$ for which $(0.1)$ holds. To the best of our knowledge, no such results are known for any compact sets in $\mathbf{C}^{N}, N>1$.

Problem 2. Let $\mu$ be a measure in $\mathbf{C}^{N}$ with $S(\mu)=\bar{B}$. Find necessary conditions on $\mu$ so that $(\bar{B}, \mu)$ satisfies (0.1).

Perhaps a better "test" set would be the totally real (closed) ball in $\mathbf{R}^{N} \subset \mathbf{C}^{N}$; cf., the discussion in [BBCL].

\section{REFERENCES}

[AT] H. Alexander and B. A. Taylor, Comparison of two capacities in $\mathbf{C}^{N}$, Math. Zeitschrift 186 (1984), 407-417. MR 85k:32034

[A] A. Ancona, Sur une conjecture concernant la capacite et l'effilement, Theorie du Potential, Orsay, Lecture Notes in Mathematics (Springer-Verlag) 1096 (1983) 34-68. MR 88f:31006

[BT1] E. Bedford and B. A. Taylor, A new capacity for plurisubharmonic functions, Acta Math. 149 (1982), 1-40. MR 84d:32024

[BT2] E. Bedford and B. A. Taylor, Plurisubharmonic functions with logarithmic singularities, Annales de l'Institut Fourier (Grenoble) 38 (1988), 133-171. MR 90f:32016

[Bl1] T. Bloom, Orthogonal polynomials in $\mathbf{C}^{N}$, Indiana Univ. Math. Journal 46 (1997), No. 2, 427-452. MR 98j:32006

[B12] T. Bloom, Some applications of the Robin function to multivariable approximation theory, J. Approx. Theory 92 (1998), 1-21. MR 98k:32021

[BBCL] T. Bloom, L. Bos, C. Christensen, N. Levenberg, Polynomial interpolation of holomorphic functions in C and $\mathbf{C}^{N}$, Rocky Mtn. J. of Math. 22 \#2 (1992), 441-470. MR 93i:32016

[C] D. Coman, Integration by parts for currents and applications to the relative capacity and Lelong numbers, to appear in Mathematica 39 (62), Academie Roumaine, Filiale de Cluj-Napoca, 1997. MR 99c:32006

[FS] J.-E. Fornaess and B. Stensones, Lectures on Counterexamples in Several Complex Variables, Princeton University Press, 1987. MR 88f:32001

[K] M. Klimek, Pluripotential Theory, Clarendon Press, Oxford, 1991. MR 93h:32021

[Ko] S. Kolodziej, The complex Monge-Ampere equation, Acta Math. 180 (1998), 69-117. CMP 98:13

[L] N. Levenberg, Monge-Ampere measures associated to extremal plurisubharmonic functions in $\mathbf{C}^{N}$, Transactions of the AMS 289 (1985), No. 1, 333-343. MR 86i:32030

[ST] H. Stahl and V. Totik, General Orthogonal Polynomials, Cambridge University Press, Cambridge, 1992. MR 93d:42029

[TZ] Nguyen Thanh Van and A. Zeriahi, Familles de polynômes presque partout borneés, Bull. Sci. Math. 107 (1983), 81-91. MR 85b:32026

[U] J. Ullman, On the regular behaviour of orthogonal polynomials, Proc. London Math. Soc. 24 (1972), 119-148. MR 45:809

[X] Y. Xing, Continuity of the complex Monge-Ampere operator, Proc. A. M. S. 124 \#2 (1996), 457-467. MR 96d:32015

[Z] A. Zeriahi, Capacité, constante de Cebysev et polynômes orthogonaux associés a un compact de $\mathbf{C}^{n}$, Bull. Sci. Math. (2) 109 (1985), 325-335. MR 87h:32039

Department of Mathematics, University of Toronto, Toronto, Ontario M5S 3G3, CANADA

E-mail address: bloom@math.toronto.edu

Department of Mathematics, University of Auckland, Private Bag 92019, Auckland, NeW ZeALAND

E-mail address: levenber@math.auckland.ac.nz 